\title{
Electromagnetically Induced Transparency
}

\author{
K. Kowalski ${ }^{1}$, V. Cao Long ${ }^{2}$, K. Dinh Xuan ${ }^{3}$, \\ M. Glódź ${ }^{1}$, B. Nguyen Huy ${ }^{3}$, J. Szonert ${ }^{1}$ \\ ${ }^{1}$ Institute of Physics, Polish Academy of Sciences, Al. Lotników 32/46, 02-668 Warsaw, Poland \\ e-mail: \{krkowal/glodz/szonj\}@ifpan.edu.pl \\ ${ }^{2}$ Institute of Physics, University of Zielona Góra, ul. Szafrana 4a, 65-516 Zielona Góra, Poland \\ e-mail:vanlongcao@yahoo.com \\ ${ }^{3}$ Faculty of Physics, Vinh University, 182 Le Duan, Vinh, Nghe An Vietnam
}

(Received: 29 June 2010; accepted: 13 September 2010)

\begin{abstract}
In the initial part of the paper, the principles of the electromagnetically induced transparency (EIT) in basic three-level schemes are sketched, and some applications of this phenomenon are described. Next a presentation follows of a five-level EIT model of Bloch equations, which was developed to reconstruct multipeak cascade-EIT spectra registered in a sample of cold ${ }^{85} \mathrm{Rb}$ atoms in MOT. The respective experiment is also described. The achieved good agreement between theory and performed experiment is documented and discussed.
\end{abstract}

Key words: electromagnetically induced transparency (EIT), cold Rb atoms in MOT, transmission spectra, multipeak EIT in a cascade scheme, semi-classical treatment, optical Bloch equations, multilevel model

\section{INTRODUCTION}

The subject of this paper is the electromagnetically induced transparency (EIT), the phenomenon belonging to the category of the coherent effects. In Sec. II we introduce the physical foundations underlying EIT. We present three basic three-level configurations of the atomic levels and fields in which EIT could be observed, $\Lambda$-, V- and cascadeschemes. Due to significant modification of the optical properties of medium, being the result of EIT, this phenomenon finds various interesting applications, especially in optics and quantum physics, but also in technology. Some of them are presented in Sec. III, with the stress on slowing, stopping and storing of light pulses. In Sec. IV we go beyond the three-level approximation. We describe our theoretical model of EIT for a multilevel cascade configuration, developed to explain the features of absorption spectra observed in our experiment with cold $\mathrm{Rb}$ atoms. The experiment was performed in Warsaw with the use of the system presented in more detail in our other paper of this issue [1]. Finally, in Sec. V we summarize the key points of this paper and draw some conclusions.

\section{PHYSICAL FOUNDATIONS OF EIT; EIT IN THREE- LEVEL SYSTEMS}

The phenomenon, which was termed electromagnetically induced transparency by Harris and co-workers [2], relies on the destructive quantum interference of the transition amplitudes, which leads to suppression of absorption or even to complete transmission of the resonant weak probe beam. EIT arises in the presence of a second (strong) laser beam coupling coherently one of the states which participate in absorption, with some other atomic state [3-6]. In Section II, EIT in the basic three-level schemes is explored.

\section{II.1. General issues}

The simplest configuration in which EIT could be observed is a three-level system as outlined in Fig. 1. Figure 1a illustrates the cascade system, in which a weak probe beam is tuned to the transition $|1\rangle \rightarrow|2\rangle$, whereas a strong coupling beam is tuned to the transition $|2\rangle \rightarrow|3\rangle$. Similarly, Fig. 1b and Fig. 1c illustrate the systems of levels and 
transitions involved in the $\Lambda$ and $\mathrm{V}$ configurations, respectively. The levels are labeled in such a way that in every configuration the transitions between the levels $|1\rangle$ and $|2\rangle$, and $|3\rangle$ are allowed electric dipole transitions, whereas the $|1\rangle \rightarrow|3\rangle$ transition is always a dipole-forbidden transition. (a)

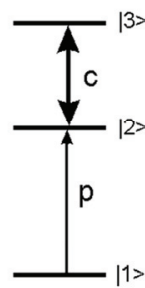

(b)

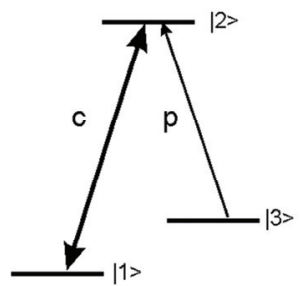

(c)

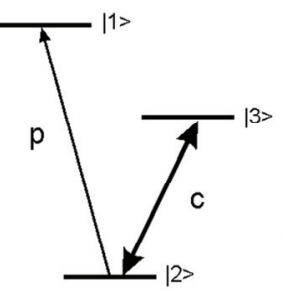

Fig. 1. The simplest configurations for EIT: (a) cascade (ladder), (b) $\Lambda$ and (c) V. The arrows denote the beams: p - probe, $\mathrm{c}-$ strong coupling

The phenomenon of EIT was predicted in 1989 [7] and experimentally observed in Sr vapor in 1991 [8]. EIT has been the subject of intensive research over the past two decades not only as a technique for stimulating transparency, but also for generating in the medium unusual dispersive properties, which accompany transparency. In Fig. 2 imaginary and real parts of the electromagnetic susceptibility $\chi\left(\omega_{p}\right)$ of the medium (collisionless atomic gas) are presented, plotted as a function of the frequency $\omega_{p}$ of the weak probe beam, relative to the atomic resonance at frequency $\omega_{p}^{0}$. For schemes in Fig 1 (a), (b) and (c) the frequency $\omega_{p}^{0}$ is to be identified with $\omega_{21}^{0}=\left(E_{2}-E_{1}\right) / \hbar$, $\omega_{23}^{0}=\left(E_{2}-E_{3}\right) / \hbar$ and $\omega_{12}^{0}=\left(E_{1}-E_{2}\right) / \hbar$, respectively, where $E_{i}$ stands for the level energy. The dashed lines in Fig. 2 are plots for the case of absence of the coupling beam, whereas the solid lines are the modified profiles in the presence of the additional strong resonant coupling beam generating EIT (see e.g., [3]). The upper plot (a) presents the imaginary part of the susceptibility $\operatorname{Im}\left[\chi\left(\omega_{p}\right)\right]$, which determines the absorptive properties of the medium (Eq. (1)). The lower plot (b) presents the real part $\operatorname{Re}\left[\chi\left(\omega_{p}\right)\right]$ related to the refractive index $n\left(\omega_{p}\right)$ (Eq. (2)),

$$
\begin{gathered}
\alpha\left(\omega_{p}\right)=k \operatorname{Im}\left[\chi\left(\omega_{p}\right)\right], \\
n\left(\omega_{p}\right)=1+\frac{1}{2} \operatorname{Re}\left[\chi\left(\omega_{p}\right)\right],
\end{gathered}
$$

where $\alpha\left(\omega_{p}\right)$ is the absorption coefficient and $k=2 \pi / \lambda$. Coefficient $\alpha\left(\omega_{p}\right)$ is related to transmission $T$ :

$$
T\left(\omega_{p}\right)=\exp \left[-\alpha\left(\omega_{p}\right) L\right]
$$

where $L$ is the length of the absorbing medium.
For plots in Fig. 2 it is assumed that the coupling beam frequency $\omega_{c}$ is tuned to $\omega_{c}^{0}$ being atomic resonance frequency for the coupling transition. In such a case, as stems from Fig. 2 (a) with relations (1) and (3), a narrow peak of increased transmission (often called "transparency window"), centered at $\omega_{p}^{0}$, develops due to EIT. Simultaneously, as follows from Fig. 2 (b) with relation (2), in the vicinity of the resonance frequency $\omega_{p}^{0}$, medium is characterized by steep and normal dispersion $\left(d n / d \omega_{p}>0\right)$ strongly depending on $\omega_{p}$ (solid line), instead of anomalous dispersion $\left(d n / d \omega_{p}<0\right)$ (dashed line) characteristic for the lack of coupling.

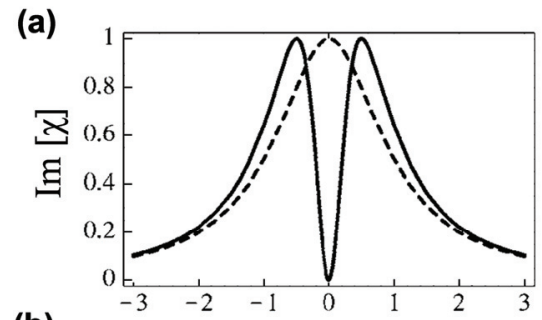

(b)

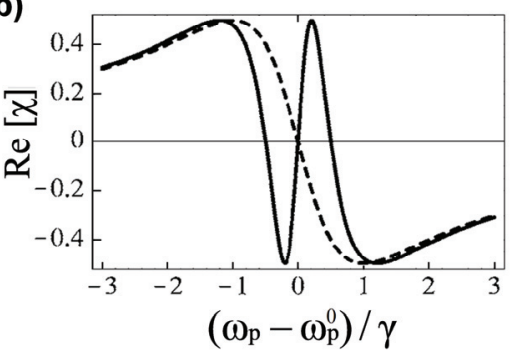

Fig. 2. Profiles of the electromagnetic susceptibility $\chi\left(\omega_{p}\right)$ of the motionless atomic gas in the vicinity of the atomic resonance $\omega_{p}=\omega_{p}^{0}$. (a) imaginary part of the susceptibility $\operatorname{Im}\left[\chi\left(\omega_{p}\right)\right]$ proportional to the absorption coefficient (formula(1)); (b) real part $\operatorname{Re}\left[\chi\left(\omega_{p}\right]\right.$ related to the refractive index (formula $\left.(2)\right)$. The dashed line shows the typical profiles of the susceptibility, whereas the solid line shows the profiles in the presence of the strong resonant coupling beam generating EIT. $\gamma$ is the radiative width of the probe resonance in the absence of coupling

There exist two ways in the literature for explaining the physical mechanism of EIT. They are equivalent under certain conditions. The first explanation. The excitation of the state $|2\rangle$ in Fig. 3 (a) can be seen as occurring via two routes (though more than two routes can also be considered) [9]. In the most simple case we have the direct rout $|1\rangle \rightarrow|2\rangle$. However, the coupling between the states $|2\rangle$ and $|3\rangle$ by the strong beam leads to an alternative pathway to achieving the state $|2\rangle:|1\rangle \rightarrow|2\rangle \rightarrow|3\rangle \rightarrow|2\rangle$. The destructive interference of the probability amplitudes for these two routes leads to the transparency for the probe beam. The second explanation. Here the dressed-atom picture is used (Fig. 3 (b). The coupling field creates a pair 
of the equivalent closely spaced dressed states $|2 d\rangle$ and $\left|2 d^{\prime}\right\rangle$. An atom in the ground state $|1\rangle$ absorbs probe photons $\hbar \omega_{p}^{0}$ (nonresonantly in the dressed-atom picture, since $\omega_{p}^{0}$ is detuned from both $|2 d\rangle$ and $\left|2 d^{\prime}\right\rangle$ states by half Rabi frequency) in two possible routes $|1\rangle \rightarrow|2 d\rangle$ and $|1\rangle \rightarrow\left|2 d^{\prime}\right\rangle$, which from the perspective of bare states lead to excitation to the bare state $|2\rangle$. The destructive interference between the transition amplitudes corresponding to the two routes results in cancelation (or at least decrease) of absorption of the probe beam, as in the first explanation $[8,10]$. (a)

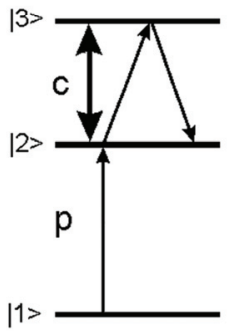

(b)

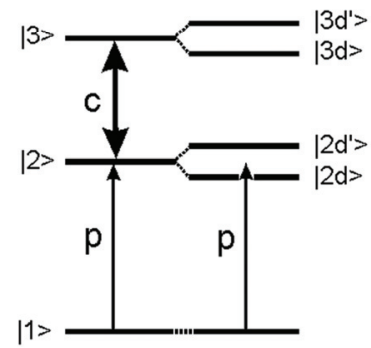

Fig. 3. Schemes for two pictures (mechanisms) of generating EIT. Cascade configuration is used as an example. Decreasing of absorption is due to the destructive interference of amplitudes for two routes leading to excitation of the state $|2\rangle$. In (a) the transitions between bare states are considered: $|1\rangle \rightarrow|2\rangle$. and $|1\rangle \rightarrow|2\rangle \rightarrow|3\rangle \rightarrow|2\rangle$; in (b) transitions to closely spaced dressedatom states are taken into account: $|1\rangle \rightarrow|2 d\rangle$ and $|1\rangle \rightarrow\left|2 d^{\prime}\right\rangle$ (see the explanations in the text)

Let us consider three ideal three-level systems of Fig. 1. Properties of EIT depend on the properties of the matrix element $\rho_{31}$, the two-photon coherence between the states $|1\rangle$ and $|3\rangle$, which are not bound by the allowed dipoletransition. All processes destroying this coherence have an impact on the effectiveness of EIT. First we assume that the relaxation is caused by the spontaneous emission only, with rates $\Gamma_{k}$.

If other relaxation processes (e.g. the dephasing collisions) are neglected, the decay rates $\gamma_{i j}$ for atomic coherences $\rho_{i j}$ are expressed by spontaneous decay rates [11]:

$$
\gamma_{i j}=\frac{\Gamma_{i}+\Gamma_{j}}{2}
$$

when dephasing processes, e.g., due to finite laser linewidths, cannot be neglected, we should add to the right-hand side of (4) the adequate dephasing relaxation rates [11].

According to (4), the decay rate $\gamma_{13}$ for the coherence $\rho_{31}$ is given by:

$$
\gamma_{13}=\frac{\Gamma_{1}+\Gamma_{3}}{2}
$$

For the configurations (a), (b) and (c) in Fig. 1, with the lowest state identified with atomic ground state, we obtain respectively:

$$
\begin{aligned}
& \gamma_{13}^{(\mathrm{a})}=\frac{\Gamma_{3}^{(\mathrm{a})}}{2}\left(\text { in the cascade system } \Gamma_{1}^{(\mathrm{a})}=0\right), \\
& \gamma_{13}^{(\mathrm{b})}=0 \quad\left(\text { in the } \Lambda \text {-system } \Gamma_{1}^{(\mathrm{b})}=0 \text { and } \Gamma_{3}^{(\mathrm{b})}=0\right), \\
& \gamma_{13}^{(\mathrm{c})}=\frac{\Gamma_{1}^{(\mathrm{c})}+\Gamma_{3}^{(\mathrm{c})}}{2}(\text { in the } \mathrm{V} \text {-system }) .
\end{aligned}
$$

From (6a)-(6c) one may conclude that the largest transparency can be obtained in $\Lambda$-system. However, other processes than spontaneous decay also influence the transparency. Such are the optical pumping of the level $|1\rangle$ by the coupling beam in $\Lambda$-scheme, and saturation of the transition $|2\rangle \rightarrow|3\rangle$ in V-scheme. A comparison of the properties of the three basic three-level configurations is performed in the papers [12-15].

In practical cases of experiments with real atoms, the very assumption of three isolated levels itself, or other idealizations could appear nonrealistic to the extent depending on the conditions of a particular experiment. For example, inhomogeneous line broadening due to atomic collisions should be taken into account for the experiments in a spectral cell at room temperature, but it may be practically neglected in the case of cold atoms in MOT. Such and similar aspects of the complexity of the problem are important to be considered by modeling the processes of EIT and by planning the experiments.

\section{II.2. Essentials of an analytical treatment of EIT in the three-level-atom approximation}

Let us return to the case of an ideal three-level scheme in its cascade implementation (Fig. 1 (a)). As above, let $\omega_{p}$ denote the frequency of the probe beam, $\omega_{21}=\omega_{p}^{0}$ the frequency at resonance for the uncoupled transition $|1\rangle \rightarrow|2\rangle$, and $\Delta_{1}=\omega_{p}-\omega_{21}$ the detuning of $\omega_{p}$ from the resonance. Similarly, let $\omega_{c}$ denote the frequency of the coupling beam, $\omega_{32}$ the frequency at resonance for the transition $|2\rangle \rightarrow|3\rangle$, and $\Delta_{2}=\omega_{c}-\omega_{32}$ the detuning. In the framework of the semiclassical approach (see Sec. IV), with the assumption of the weak probe beam, the expressions for the matrix elements of the density operator in the stationary regime have been obtained e.g., in [13]. The weak probe beam solution for the matrix element $\rho_{21}$ related to absorption and dispersion reads:

$$
\rho_{21}=-E_{p} \frac{i \mu_{21} / 2 \hbar}{\gamma_{21}-i \Delta_{1}+\frac{\Omega_{c}^{2}}{\gamma_{31}-i\left(\Delta_{1}+\Delta_{2}\right)}},
$$


where $\Omega_{c}=\mu_{32} E_{\mathrm{c}} / \hbar$ is the Rabi frequency from the coupling field, $\mu_{21}$ and $\mu_{32}$ are the matrix elements of the dipole moment operator for the appropriate transitions, $E_{p}\left(E_{c}\right)$ is the amplitude of the probe (coupling) field. For the considered configuration in which the state $|1\rangle$ is the ground state, the decoherence rates $\gamma_{21}$ and $\gamma_{31}$ are (according to formula (4)) $\Gamma_{2} / 2$ and $\Gamma_{3} / 2$, respectively. The complex susceptibility $\chi\left(\omega_{\mathrm{p}}\right)$ is related to $\rho_{21}$ by polarization $P$ of the atomic medium, i.e. the linear polarization with respect to $E_{p}$

$$
\begin{gathered}
P=\frac{1}{2} \varepsilon_{0} E_{p}\left[\chi\left(\omega_{p}\right) e^{-i \omega_{p} t}+c . c .\right]= \\
=-\mu_{21} N \rho_{21} e^{-i \omega_{p} t}+c . c .,
\end{gathered}
$$

where $N$ is the atomic density. Because of the nonlinearity due to the strong coupling field, $\chi\left(\omega_{p}\right)$ is often called the dressed linear susceptibility (e.g., [6]). From the formulas (7) and (8) we obtain the expression for $\chi$ :

$$
\chi=\frac{i \mu_{21}^{2} N / \hbar \varepsilon_{0}}{\gamma_{21}-i \Delta_{1}+\frac{\Omega_{c}^{2}}{\gamma_{31}-i\left(\Delta_{1}+\Delta_{2}\right)}} .
$$

According to considerations in Sec II.1, the imaginary and real parts of the susceptibility $\chi$ in formula (9) describe respectively the dissipative (absorption) and dispersive properties of the medium under the conditions of EIT, see formulas (1) and (2). Plots of $\operatorname{Im}[\chi]$ and $\operatorname{Re}[\chi]$ (with some realistic parameters assumed in (9) as a function of probe detuning $\Delta_{1}$, and with the detuning $\Delta_{2}$ fixed at 0 value, would resemble plots in Fig. 2. For a nonresonant coupling $\left(\left|\Delta_{2}\right| \neq 0\right)$, but providing $\omega_{c}$ is in the vicinity of the resonance, the EIT dip can be still observed, but it gradually deforms with $\left|\Delta_{2}\right|$. For the cascade EIT scheme, the absorption minimum appears providing that the two detunings satisfy condition for the two-photon resonance:

$$
\Delta_{1}+\Delta_{2}=0 .
$$

For each of the configurations $\Lambda$ or $\mathrm{V}$ the minimum appears for

$$
\Delta_{1}-\Delta_{2}=0,
$$

what follows from a similar expression for $\chi$, as (9).

\section{APPLICATIONS OF EIT}

The phenomenon of EIT gives us the possibility to modify, in a controlled way, the optical response of an atomic medium, therefore it finds numerous applications. In many cases a three-level plus two-field model can be considered to be a fairly good approximation of the physical situation. Out of the schemes drawn in Fig. 1, the $\Lambda$-configuration is perhaps most often used. Combinations of the basic schemes are also applied.

A number of selected applications of EIT are presented in Sec. III.1, while Sec. III.2 is entirely devoted to one topic, namely to the modification of group velocity of light, which may even lead the extreme of "stopping" or "storing" light pulses.

\section{III.1. Some applications of EIT}

The significant change in transmission of the beam propagating through the medium by turning on another beam has made EIT attractive in view of its prospect applications in telecommunication. In this respect, usefulness of transient EIT to optical switching is investigated. The results of basic research in this field have been presented by Chen et al. for cold $\mathrm{Rb}$ atoms [16]. The shapes of the pulses of the transmitted probe beam, generated as a result of switching-on the coupling beam in EIT $\Lambda$-system, were registered and theoretically analyzed. This switching-on had a nonadiabatic character - it lasted shorter than both the relaxation times and the inverse of the Rabi frequency. A periodic optical switching in the cascade scheme of EIT was demonstrated and discussed in another paper [17] for the medium of $\mathrm{Rb}$ atomic vapor at room temperature. Toggling on an off the infrared probe beam (tuned to the resonance $5 \mathrm{~S}_{1 / 2} \rightarrow 5 \mathrm{P}_{3 / 2}$ ) was executed by the visible coupling beam (tuned to the resonance $5 \mathrm{P}_{3 / 2} \leftrightarrow 8 \mathrm{D}_{5 / 2}$ ), modulated in amplitude. Harris and Yamamoto demonstrated a model of optical switching in a four-level scheme with three laser beams [18]. The authors supplemented the classical scheme of $\Lambda$-type EIT with an additional coupling, which introduced decoherence, and thus eliminated the transmission window.

The influence of the magnetic field on the position of the atomic Zeeman sublevels $m_{F}$ leads to an important application of EIT: in the magnetometry. In this case the strong change of the refractive index near the EIT resonance at $\omega_{p}^{0}$ is employed. Scully and Fleischhauer et al. proposed a based on EIT method of the optical detection of magnetic fields with very high sensitivity $[19,20]$. Let us assume that the change of the magnetic field $B$ results in a change of $\omega_{p}^{0}$. Under EIT it may cause substantial change of the refractive index $n$ and a big $d n / d B$ value, which provides the high sensitivity to the method. The change in $n$ is being detected by a comparative interferometric method. The sensitivity of a magnetometer of 
this kind is of the order of $10^{-15} \mathrm{~T}$ and is comparable to the sensitivity of supersensitive SQUID magnetometers [21] (SQUID stands for Superconducting Quantum Interference Device).

Though in this paper we concentrate on EIT in free atoms, it is due to mention the fast growing interest in EIT and related coherent phenomena in solid state media. Results of basic and/or applied research in this field have been reported for rare earth doped insulators [22, 23], semiconductor quantum wells and quantum dots [24-31], optical fibres [32], silicon ring resonators [33] photonic crystals and photonic crystal waveguides [34, 35].

An application of EIT in manipulating signals in the optical fiber communication systems was presented by Rostami et al. [36]. The authors proposed to make use of EIT generated in semiconductor materials to tune the optical filters based on ring-resonator. There also exist reports about EIT in the semiconductor quantum wells, whose spectral properties could be controlled electronically (e.g., [37, 38]).

More about relevant applications of solid state materials can be found in some papers referenced at the end of Sec. III.2.

\section{III.2. Slow, ultraslow and stopped light pulses}

The fact that along with EIT, the refractive index of the medium $n\left(\omega_{p}\right)$ may considerably change in the vicinity of the resonance $\omega_{p}^{0}$, makes up foundations for slowing down light, in the sense of spectacular reduction of its group velocity $[3,39]$.

The group velocity is given by the formula:

$$
v_{g}=\frac{c}{n\left(\omega_{p}\right)+\omega_{p} \frac{d n}{d \omega_{p}}},
$$

where $c$ is the vacuum speed of light.

From the formulas (11) and (2), and from Fig. 2 (b), where steepness of the slope of $\operatorname{Re}[\chi]$ about $\omega_{p}^{0}$ is shown, one can deduce that the probe pulse at the frequency $\omega_{p}^{0}$ should dramatically slow down under the conditions of EIT. This was first pointed out by Harris et al. [40].

In the first experiments the reduction of the group velocity of light by a factor of $c a 10^{2}-10^{3}$ was achieved. For example, Kasapi et al. obtained the velocity $v_{g}=c / 165$ in lead vapor [41], and Schmidt's group obtained $v_{g}=c / 3000$ in Cs vapor [42]. Hau et al. were the first to obtain extremely small group velocity in Bose-Einstein condensate of $\mathrm{Na}$ atoms. The sodium atoms were cooled to the temperature varied in the range $2.5 \mu \mathrm{K}-50 \mathrm{nK}$ (the critical temperature for obtaining BEC of Na atoms is $T_{c}=435 \mathrm{nK}$ ) and EIT was generated at different powers of the coupling beam. According to theoretical predictions, the group velocity decreases with decreasing coupling power and with increasing density of the atomic cloud. The lowest group velocity $17 \mathrm{~m} / \mathrm{s}$, corresponding to $v_{g}=c / 10^{7}$, was achieved in this condensate at the temperature of $50 \mathrm{nK}$ [43]. Soon, in the same year 1999, similarly small $v_{g}$ value was reported by Kash et al. [44], who reached $v_{g}=90 \mathrm{~m} / \mathrm{s}$ for "warm" ${ }^{87} \mathrm{Rb}$ atoms in a cell at the temperature of $360 \mathrm{~K}$. Also in the same year, Budker et al., obtained $v_{g}=8 \mathrm{~m} / \mathrm{s}$ for ${ }^{85} \mathrm{Rb}$ at room temperature [45].

When a pulse of the probe light enters a medium characterized by decreased group velocity, it becomes spatially compressed in the propagation direction. This is due to the fact that the front of the pulse in the "slowing down medium" propagates much more slowly than its back end which has not yet reached the medium. The pulse is spatially compressed by the factor $c / v_{g}$ (with $c$ being the speed outside the medium), therefore, with a big change in velocity, even very long pulses could completely fit into as small media as MOT or BEC (Bose-Einstein condensate). Slowing down and compression of the light pulses to the size of a medium made it possible to "imprison" the pulses in the medium.

In 2001, Philips et al. [46] and Liu et al. [47] demonstrated experimentally "stopping of light". The latter group managed to stop the light pulses for up to $1 \mathrm{~ms}$ in $\mathrm{Na}$ atoms at the temperature of $c a 1 \mu \mathrm{K}$ in a magnetic trap. The velocity of the light pulse was lowered by using EIT in $\Lambda$-configuration. Next, when the compressed light pulse was already localized within the sample of $\mathrm{Na}$ atoms, the coupling beam was suddenly switched off for the time of $1 \mathrm{~ms}$, which caused "freezing" the coherent information, initially contained in the laser fields, into atoms. Thus the information about the amplitude and phase of the pulse became stored. After $1 \mathrm{~ms}$ the coupling laser was turned back on and the probe pulse was regenerated due to interaction of the coupling beam with the atoms. The form of the pulse which left the medium was the same, as it was at the input. The maximum time of the "storage" is limited by the dissipative processes which destroy the coherence. It is due to mention that in view of the mechanisms described above, the terms "imprisonment", "stopping" and "storing" of the light pulse used to describe what happens between switching off and switching on the coupling beam, have only a symbolic meaning. This described process can be used to transfer information via quantum methods. For example, in the time when the information about the pulse is stored in the atoms, one can modify it by acting on atoms with magnetic or electric fields. On regenerating the pulse, the modified information is being transferred to the pulse, and next carried out by the "released" pulse. 
Since the early works at the end of the past and at the beginning of the present century, investigations in the field of slowing down (but also accelerating) and stopping light pulses have been constantly progressing; beside atoms, also solids have drown attention as slowing media. We provide references to a selection of relevant theoretical and experimental papers, some of them are reviews, see works [3, 4859] and references therein. Below we mention one of the cited experiments.

In 2007, Ginsberg, Garner and Hau published results of a very interesting experiment [55], in which a slow light pulse was stopped and "stored" in one of two BoseEinstein condensates, and next the pulse was recreated from the second BEC, placed initially at almost macroscopic distance from the first condensate $(160 \mu \mathrm{m})$. Information from one to the other BEC was transferred by a material wave.

\section{FIVE-LEVEL MODEL FOR A CASCADE SCHEME OF EIT IN COLD ATOMS OF RUBIDIUM 85}

In Section IV we deal with a case of multilevel EIT. Authors' investigations concerning EIT in a cascade scheme, with dense hyperfine structure in the uppermost level, are described. Because of the proximity of neighboring $h f s$ substates, which all participate in coupling, a three-level approximation is not legitimate in this case.

We introduce a five-level model (with five levels and two beams: probing and coupling), developed to explain multiple windows of EIT registered in a cascade scheme $5 \mathrm{~S}_{1 / 2}(F) \rightarrow 5 \mathrm{P}_{3 / 2}\left(F^{\prime}\right) \rightarrow 5 \mathrm{D}_{J}{ }^{\prime},\left(\left\{F^{\prime \prime}\right\}\right)$ of cold ${ }^{85} \mathrm{Rb}$ atoms in MOT. First, in Sec IV.1 we describe details of the applied scheme(s), and performed measurements. Two examples of registered transmission spectra with pronounced multiple EIT peaks are presented. An outline of the model is given in Sec. IV.2.1. The spectra of transmission of the probe beam tuned in the vicinity of a $h f s F \rightarrow F^{\prime}$ resonance were modeled as depending on the coupling beam intensity or on the coupling beam frequency-detuning from a $F^{\prime} \leftrightarrow F^{\prime \prime}$ resonance. They are compared with those obtained experimentally and discussed in Sec. IV.2.2. The experimental work was performed in Warsaw by using the system described in our other paper of this issue [1], as well as in [61-63]. Section IV is partially based on references [63, 64].

We have been interested in multiple transparency windows with regard to the potential use of the scheme to simultaneous slowing down of light pulses at various frequencies [65]. Conclusions concerning this issue are drawn in Sec. V.

\section{IV.1. Details of the applied scheme for generating multiple transparency windows}

In a basic EIT scheme with three levels and two fields, as in Fig. 1, a single peak of enhanced transmission is expected, as analyzed in Sec. II. As stated above, one way to produce multiple transparency windows is to involve in the scheme a manifold of close lying atomic sublevels, which could be simultaneously coupled by a single monochromatic coupling beam [65]. This was the method adopted in our experiments and it is out of the scope of this paper to analyze other methods. However, for a short survey of the ones published in articles [66-69], we refer to [63].

The curly brackets enclosing $F^{\prime \prime}$ in the process $5 \mathrm{~S}_{1 / 2}(F)$ $\rightarrow 5 \mathrm{P}_{3 / 2}\left(F^{\prime}\right) \rightarrow 5 \mathrm{D}_{J}$ ' $\left(\left\{F^{\prime \prime}\right\}\right)$ symbolize a set of levels. For

${ }^{85} \mathrm{Rb}$ atoms, under conditions of our experiment, up to three hfs $F^{\prime \prime}$ states can be simultaneously excited (as follows from the selection rules for electric-dipole transitions), at moderate values of Rabi frequency of the coupling transition. Partial scheme of ${ }^{85} \mathrm{Rb}$ levels for $J "=5 / 2$ is visualized in Fig. 4. Transitions are also marked. The cases in Fig. 4 (a) and (b) differ by the ground state hfs component used as lower state for the probe beam $P$ transition. The broken fragments of the arrows $P$ indicate the tuning ranges of beam $P$. The ranges are different for (a) and (b).

In Fig. 4 (a), as well as in (b), three groups of allowed resonant transitions, connecting the $h f s$ components of the states $5 \mathrm{P}_{3 / 2}$ and $5 \mathrm{D}_{5 / 2}$, are marked. These are groups $\mathrm{C}_{2}, \mathrm{C}_{3}$, $\mathrm{C}_{4}$ for (a) and $\mathrm{C}_{1}, \mathrm{C}_{2}, \mathrm{C}_{3}$ for (b). For each group, the adjacent $5 \mathrm{D}_{5 / 2}\left(F^{\prime \prime}\right) h f s$ sublevels are separated by less than $10 \mathrm{MHz}$. Therefore, in each group $\mathrm{C}_{i}$, all three $F_{i_{k}}$ states can be simultaneously coupled to their proper $F_{i}^{\prime}$ state, by a single-frequency laser beam (not present in Fig. 4) at sufficient intensity to provide Rabi frequency comparable to the $h f s$ splitting. For simplicity, in the text below we use the same symbol $\mathrm{C}_{i}$ to designate a marked group of allowed transitions, the coupling beam at a frequency fixed in the vicinity of the relevant group $\mathrm{C}_{i}$, as well as the triple coupling caused by this beam. Obviously, each of the three elementary $F_{i}^{\prime} \leftrightarrow F_{i_{k}}^{\prime \prime}$ couplings of the coupling $\mathrm{C}_{i}$ is characterized with different Rabi frequency, as well as with different detuning of the laser beam frequency from the (unperturbed) $F_{i}^{\prime} \leftrightarrow F_{i_{k}}^{\prime \prime}$ resonance.

Arrows $T$ and $R$ stand, respectively, for the trapping and repumping transitions of MOT. Trapping and repumping beam powers and detunings were selected for minimizing their influence on the experiment, and still preserving stable MOT operation.

In Fig. 4, two $\mathrm{C}_{i}$ groups of allowed transitions are distinguished, with thickened arrows and with enhanced $\mathrm{C}_{i}$ 
(a)
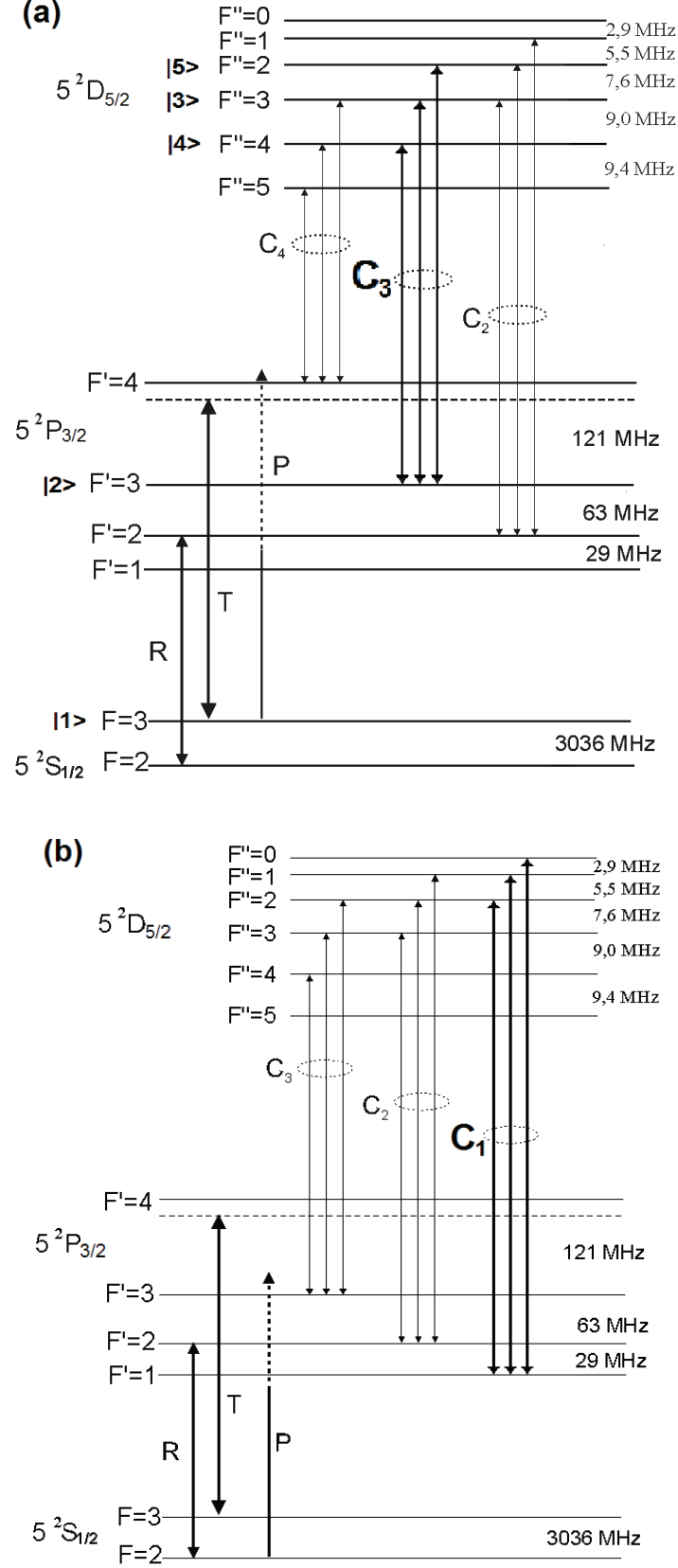

Fig. 4. The partial scheme of levels of the ${ }^{85} \mathrm{Rb}$ atom, in which EIT in cascades $5 \mathrm{~S}_{1 / 2}(F) \rightarrow 5 \mathrm{P}_{3 / 2}\left(F^{\prime}\right) \rightarrow 5 \mathrm{D}_{5 / 2}\left(\left\{F^{\prime \prime}\right\}\right)$ can be generated. The ground state for probe beam $P$ absorption is the $F=3$ state (a) or $F=2$ (b). The dashed part of the arrow denotes the frequency range in which $P$ is tuned, in respect to the allowed transitions in the first step of a cascade. $\mathrm{C}_{i}$ are groups of allowed resonant transitions in the second step of a cascade. Values of index $i$ are 2, 3 or 4 for (a) and 1,2 or 3 for (b). Due to dense hfs structure of $F$ " levels, a single coupling beam (not marked in the figures) at a frequency fixed in the vicinity of frequencies of a group $\mathrm{C}_{i}$ can simultaneously couple the three $F_{i}^{\prime \prime}$ states to their proper $F_{i}$ state. The groups $\mathrm{C}_{3}$ in (a) and $\mathrm{C}_{1}$ in (b) correspond to couplings for EIT spectra shown in Fig. 5 (a) and (b), respectively. The experiment is performed in a sample of cold ${ }^{85} \mathrm{Rb}$ atoms in MOT; $T$ is for the trapping- and $R$ for the repumpingtransition. The numbers in ket symbols in (a) indicate the correspondence between the ${ }^{85} \mathrm{Rb}$ levels and the levels numbered in the five-level model of Sec. IV.2.1 (Fig. 6) symbols: $\mathrm{C}_{3}$ in Fig. 4 (a), and $\mathrm{C}_{1}$ in Fig 4 (b). Two corresponding exemplary spectra of registered probe transmission with couplings $\mathrm{C}_{3}$ and $\mathrm{C}_{1}$ are shown in Fig. 5 (a) and (b), respectively. The approximate frequency of the $\mathrm{C}_{3}$ $\left(\mathrm{C}_{1}\right)$ laser beam, relative to uncoupled $F^{\prime} \leftrightarrow F^{\prime}$ ' resonance, is given in the Fig. 4 caption. For each spectrum (in black line) the coupling beam power was $I_{c}=400 \mathrm{~mW} / \mathrm{cm}^{2}$ (upper limit for our experiments); the spectrum taken with the coupling laser off $\left(I_{c}=0 \mathrm{~mW} / \mathrm{cm}^{2}\right.$, grey weak line $)$ is also given for reference. In spectrum (a) (spectrum (b)) transmission dips due to probe absorption from the $F=3$ $(F=2)$ state to $F$ ' states with quantum numbers: 2, 3, 4 (1, $2,3)$ can be seen. The dip due to absorption to the coupled state (coupling $\mathrm{C}_{3}(\mathrm{C} 1)$ ) is broadened and engraved with
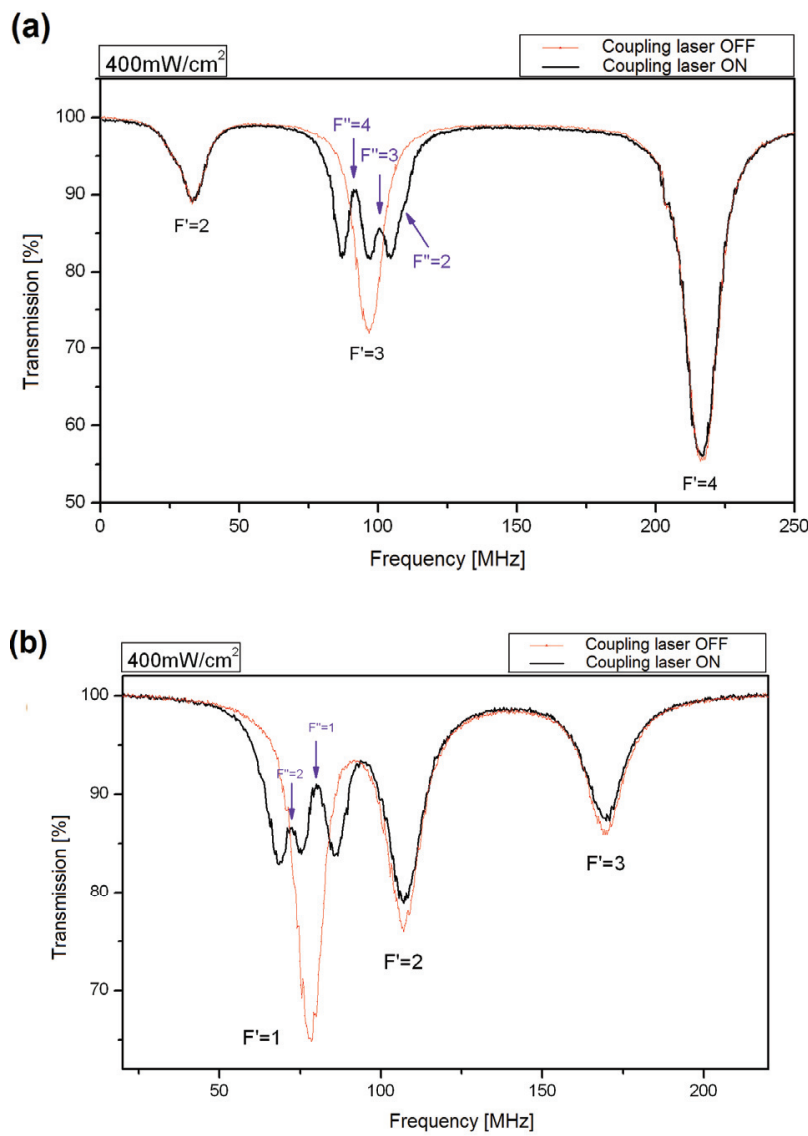

Fig. 5. Two examples of probe transmission spectra (black lines) registered in EIT experiments for cold ${ }^{85} \mathrm{Rb}$ atoms in MOT. The probe is tuned across the transitions $5 \mathrm{~S}_{1 / 2}(F=3) \rightarrow 5 \mathrm{P}_{3 / 2}\left(F^{\prime}=2,3\right.$, 4) (a) or $5 \mathrm{~S}_{1 / 2}(F=2) \rightarrow 5 \mathrm{P}_{3 / 2}\left(F^{\prime}=1,2,3\right)$ (b). The strong beam couplings: for spectrum (a) the level $5 \mathrm{P}_{3 / 2}\left(F^{\prime}=3\right)$ is coupled to the three $5 \mathrm{D}_{5 / 2}\left(F^{\prime \prime}=4,3,2\right)$ levels or for spectrum (b) the level $5 \mathrm{P}_{3 / 2}\left(F^{\prime}=1\right)$ is coupled with the three $5 \mathrm{D}_{5 / 2}\left(F^{\prime \prime}=3,2,1\right)$ levels (see Fig. 4). The frequency of the coupling laser is set close to the center of $\mathrm{C}_{3}$ (a), or between resonances $F^{\prime}=1 \leftrightarrow F^{\prime \prime}=2$ and $F^{\prime}=1 \leftrightarrow F^{\prime \prime}=1$ (b). For reference, spectra registered with the coupling beam off are also given (grey weak lines). Zerodetunings are chosen arbitrarily 
the EIT features of enhanced transmission due to the $h f_{s}$ components $F "=4,3,2(F "=2,1,0$; though here the last peak is hard to be observed of the $5 \mathrm{D}_{5 / 2}$ state. The separations between the EIT peaks are close to the spacings between respective $h f s$ components.

Besides these presented examples, we have registered EIT spectra for most $\mathrm{C}_{i}$ couplings related to Fig. 4 , as well as for analogous ones with $J^{\prime \prime}=3 / 2$, i.e., for the $5 \mathrm{D}_{3 / 2}$ state in the role of the uppermost state. The quality of multiple EIT spectra in the schemes with $J^{\prime \prime}=5 / 2$ appeared comparably good as this of examples in Fig. 5. The multiple EIT peaks in the spectra, registered at the same laser power, for the schemes to $5 \mathrm{D}_{3 / 2}$, were far less pronounced, due to lower transition probabilities and less dense hfs structure of $J "=3 / 2$ fs component.

Spectra were typically registered under conditions of operating MOT; such are the ones presented in Fig. 5, and the experimental ones in Fig. 7. Some measurements were also done during short periodic breaks in MOT operation (with MOT fields switched off), followed by much longer periods of rebuilding MOT (with MOT fields switched on), see experimental spectra in Fig. 8. With the intention to compare our results with those of the work [65], from here on we are interested only in the spectra referring to the case of $\mathrm{C}_{3}$ coupling, exemplified in Fig. 5 (a). i.e. the ones corresponding to the process $5 \mathrm{~S}_{1 / 2}(F=3) \rightarrow 5 \mathrm{P}_{3 / 2}\left(F^{\prime}=3\right)$ $\leftrightarrow 5 \mathrm{D}_{5 / 2}\left(F^{\prime \prime}=4,3,2\right)$. In Sec. IV.2 we report the theoretical calculations aimed at reproducing the spectra registered at gradually varied experimental conditions.

\section{IV.2. The theoretical model compared with the experimental results}

\section{IV.2.1. The outline of the model}

As mentioned above, a five-level model of optical Bloch equations was developed, with two fields $\mathrm{P}$ and $\mathrm{C}$ at optical frequencies (see Fig. 6), adopted to the experimental conditions of EIT in $5 \mathrm{~S}_{1 / 2}(F=3) \rightarrow 5 \mathrm{P}_{3 / 2}\left(F^{\prime}=3\right) \leftrightarrow 5 \mathrm{D}_{5 / 2}$ $\left(F^{\prime \prime}=4,3,2\right)$ scheme for cold ${ }^{85} \mathrm{Rb}$ atoms [63, 64]. The model is similar to the one presented in Ref. [65].

The states of the model are assigned to the real states of the ${ }^{85} \mathrm{Rb}$ atom, as visualized by numbering the relevant states in Fig. 4 (a) with the same numbers in ket-symbols, as in Fig. 6. The arrow P in Fig. 6 represents the weak probe beam at the frequency $\omega_{p}$, which excites the transition $|1\rangle \rightarrow|2\rangle$. The strong coupling beam $\mathrm{C}$ at the frequency $\omega_{c}$ couples the level $|2\rangle$ with the group of three closely spaced levels $|4\rangle,|3\rangle$ and $|5\rangle$ separated by $\delta_{1}$ and $\delta_{2}$, respectively. We define the beam frequency detunings as:

$$
\Delta_{c}=\omega_{c}-\omega_{32}, \quad \Delta_{p}=\omega_{p}-\omega_{21},
$$

where $\omega_{32}$ and $\omega_{21}$ are frequencies at respective resonances.

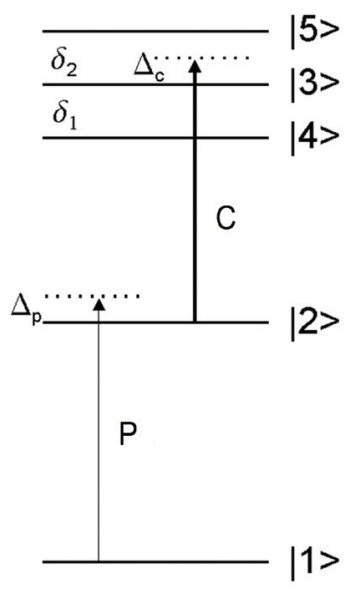

Fig. 6. The scheme for our five-level model, as described in the text

Evolution equation for the density operator $\rho$ [71] for the system of a five-level atom interacting with two laser fields, in dipole- and RWA-approximations, takes the form:

$$
\frac{\partial \rho}{\partial t}=-\frac{i}{\hbar}[H, \rho]+\Lambda \rho
$$

where the Hamiltonian $H$ is the sum of the unperturbed atomic Hamiltonian $H_{\text {at }}$ and the interaction Hamiltonian $H_{\text {int }}$,

$$
H=H_{\text {at }}+H_{\text {int }},
$$

and the term $\Lambda \rho$ accounts for the relaxation mechanisms of the system, characterized by decay parameters.

The Hamiltonians $H_{\text {at }}$ and $H_{\text {int }}$, in the basis of the five states of the model are expressed as:

$$
\begin{gathered}
H_{\mathrm{at}}=-\hbar \Delta_{p} \sigma_{22}-\hbar\left(\Delta_{p}+\Delta_{c}\right) \sigma_{33}+ \\
-\hbar\left(\Delta_{p}+\Delta_{c}+\delta_{1}\right) \sigma_{44}-\hbar\left(\Delta_{p}+\Delta_{c}-\delta_{2}\right) \sigma_{55}, \\
H_{\mathrm{int}}=-\hbar \Omega_{21} \sigma_{21}-\hbar \Omega_{32} \sigma_{32}+ \\
-\hbar \Omega_{42} \sigma_{42}-\hbar \Omega_{52} \sigma_{52}+H . c .
\end{gathered}
$$

where $\sigma_{i j}=|i\rangle\langle j|(i, j=1 . .5), \Delta_{p}$ and $\Delta_{c}$ are defined as in (12), and respective Rabi frequencies are:

$$
\begin{gathered}
\Omega_{p} \equiv \Omega_{21}=\mu_{21} E_{p} / \hbar, \\
\Omega_{k 2}=\mu_{k 2} E_{c} / \hbar,
\end{gathered}
$$


with $k=3,4,5$, and with $E_{p}\left(E_{c}\right)$ being the electric field amplitude of the probe (coupling) beam, and $\mu_{i j}$ respective transition matrix elements. We express $\Omega_{42}$ and $\Omega_{52}$ by $\Omega_{c} \equiv \Omega_{32}$ :

$$
\Omega_{42}=a_{42} \Omega_{c}, \quad \Omega_{52}=a_{52} \Omega_{c},
$$

where $a_{i j}$ are relative values:

$$
a_{42}=\frac{\mu_{42}}{\mu_{32}}, \quad a_{52}=\frac{\mu_{52}}{\mu_{32}} .
$$

Equation (13), written in the form of a set of equations for density matrix elements $\rho_{i j}=\langle i|\rho| j\rangle(i, j=1 . .5)$, was numerically solved by assuming stationary regime $\left(\dot{\rho}_{i j}=0\right)$ and realistic parameters corresponding to the conditions of our modeled experiment.

The density matrix element $\rho_{21}\left(\omega_{p}\right)$, result of calculations, was used to compare the theory with the experiment by using relations disclosed in Sec. II. According to formulas (1) and (8), $\rho_{21}\left(\omega_{p}\right)$ is related to the absorption coefficient $\alpha\left(\omega_{p}\right)$ via the dressed linear susceptibility $\chi$,

$$
\alpha\left(\omega_{p}\right)=k \operatorname{Im}\left[\chi\left(\omega_{p}\right)\right]=-\frac{2 \mu_{21} N k}{\varepsilon_{0} E_{p}} \operatorname{Im}\left[\rho_{21}\left(\omega_{p}\right)\right],
$$

(while the dispersion is related to $\left[\operatorname{Re}\left(\rho_{21}\right)\right]$, see formulas (2) and (8)). As defined in Sec. II, $N$ is the number density of the (cold in our case) atoms; $k=\omega_{p} / c ; \varepsilon_{0}$ is the vacuum permittivity. In the experiment, we registered transmission spectrum $T\left(\omega_{p}\right)$. This is related to $\alpha\left(\omega_{p}\right)$ via (3): $T\left(\omega_{p}\right)=\exp \left[-\alpha\left(\omega_{p}\right) L\right]$ (where $L$ is the length of the absorbing medium), and thus to $\operatorname{Im}\left[\rho_{21}\left(\omega_{p}\right)\right]$, through relation (20).

\section{2.2. Comparison: theory-experiment}

In this section a selection of results is presented and discussed. In Figures 7 and 8 the theoretical EIT spectra (right column) are compared with the relevant experimental ones (left column). The probe beam was scanned in the vicinity of the $5 \mathrm{~S}_{1 / 2}(F=3) \rightarrow 5 \mathrm{P}_{3 / 2}\left(F^{\prime}=3\right)$ transition. The frequency axis displays detuning $\left(\Delta_{p}\right)$ from the exact resonance in the absence of the coupling field. In the course of spectrum registration, the coupling field frequency was fixed, detuned by a $\Delta_{c}$ value from the $5 \mathrm{P}_{3 / 2}\left(F^{\prime}=3\right) \leftrightarrow 5 \mathrm{D}_{5 / 2}$ $\left(F^{\prime \prime}=3\right)$ resonance.

The dependence of the EIT spectra on the couplingbeam intensity $I_{c}$, or equivalently on Rabi frequency $\Omega_{c}$, is shown in Fig. 7. Both the experimental and assumed in calculations detunings $\Delta_{c}$ are given. For most of the spectra, $\Delta_{c}$ is close to $-8 \mathrm{MHz}$.

In Fig. 8, there are the spectra with two different detunings: $\Delta_{c}=+8 \mathrm{MHz}$ (upper ones) and $\Delta_{c}=-10 \mathrm{MHz}$ (lower ones). Both experimental spectra were registered at $I_{c}=400 \mathrm{~mW} / \mathrm{cm}^{2}$, corresponding to Rabi frequency $\Omega_{c}=7 \mathrm{MHz}$. Unlike the spectra in Fig. 7, obtained with operating MOT, these were registered in optical molasses, with MOT beams temporary switched off. Because of some details of the measurement procedure, the spectra in Fig. 8 are more noisy and the EIT features appear to be more shallow than the ones in Fig. 7 corresponding to the same $I_{c}$ value.

In the theoretical simulations based on the five-level model presented above, the value for the parameter $\Omega_{c} \equiv \Omega_{32}$ (Rabi frequency) has to be assumed, (and from $\Omega_{c}$, the values for $\Omega_{42}$ and $\Omega_{52}$ are derived, since they are taken to be bound to $\Omega_{c}$, by relations (18)). Within the model, a single value for $\Omega_{c}$ (respective to given conditions of the experiment) is required. However, it is not a priori obvious that the real physical situation of our experiment, can be described by a single, effective Rabi frequency value, which would be representative for all elementary acts, of (nonlinear) atomcoupling-field interactions across MOT, as well as for the whole period of registration. This doubt comes from the fact, that many simplifications and idealizations are inherent to our model. Let us list only two of them. (i) The model does not take into account the presence of quasi-degenerate magnetic substates with the quantum number $m_{F}$. That is, in the real atom, the polarized light is coupled to a $\left(F^{\prime}, m_{F^{\prime}}\right) \leftrightarrow\left(F^{\prime \prime}, m_{F^{\prime \prime}}\right)$ transition, and not merely to a $F^{\prime} \leftrightarrow$ $F$ " transition. Every such coupling should in fact be characterized by its own $\Omega$ value which requires a new model, largely enhanced (it should be also noted that the probe beam polarization and magnetic states in the first step are disregarded as well) [72]. (ii) The model does not account for nonuniformity in $E_{c}$ in the region of interaction, as well as for fluctuations in $E_{c}$ during the time of registration.

Nevertheless, in practice the assumption of an effective $\Omega_{c}$, appeared successful. In the process of matching the theoretical spectra to the relevant experimental ones, a value of an effective $\Omega_{c}$, was attributed to each spectrum registered at a given intensity $I_{c}$. The values of $\Omega_{c}$ were taken to be proportional to (mean) $E_{\mathrm{c}}$ values (thus to $\sqrt{I_{c}}$ ). The common proportionality factor was optimized to obtain the best match of theoretical and experimental EIT features, with regard to the whole series of experimental spectra, such as the series in Fig. 7.

As can be noticed in Figures 7 and 8, the overall width of each calculated absorption profile exceeds such a width of the corresponding experimental absorption profile. It seems that too big a value was assumed in the model for the rate of excitation decay for the state $|2\rangle$. The rate is related to the width of the absorption profile with the cou- 

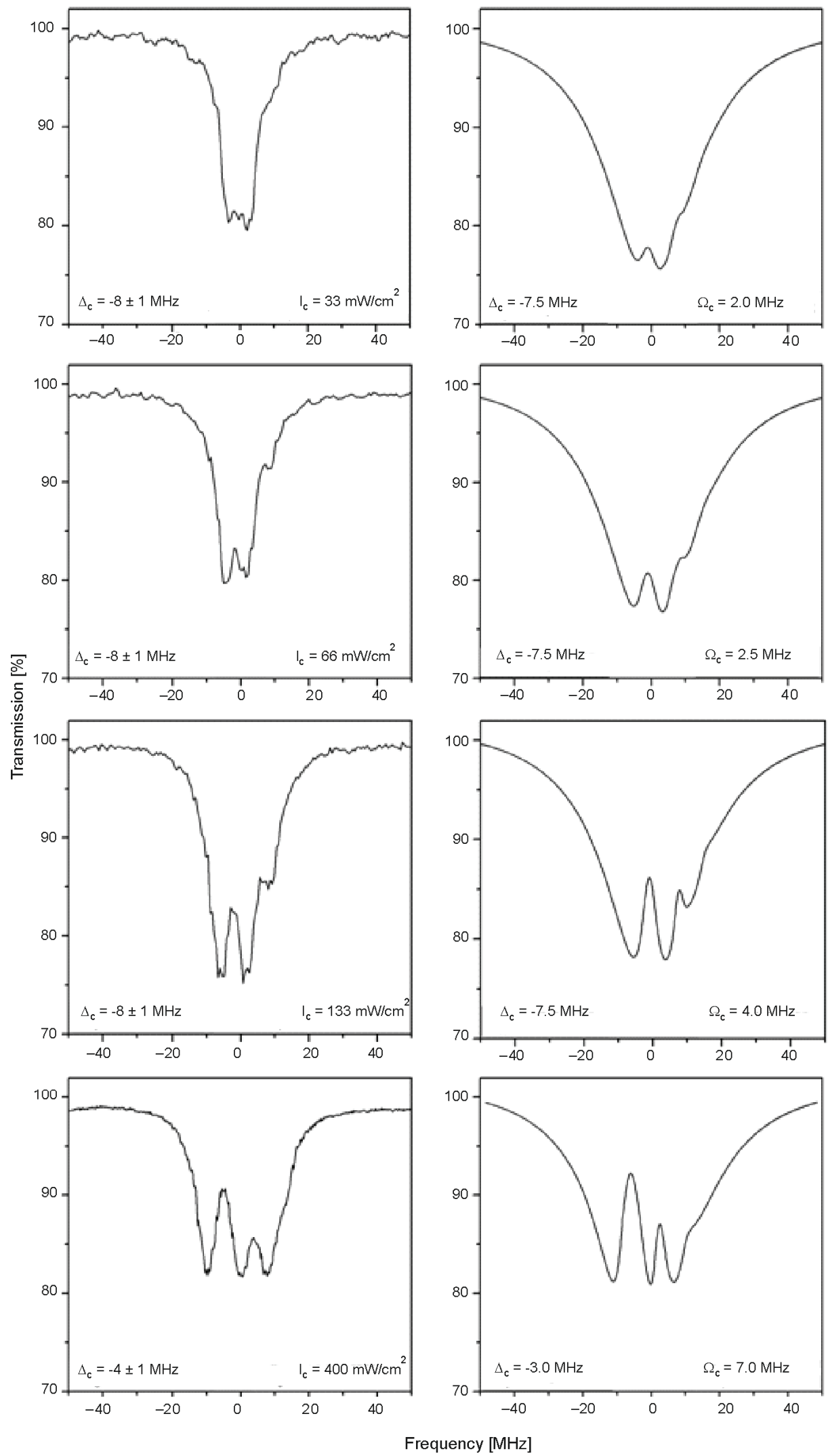

Fig. 7. EIT spectra: theoretically simulated (right column) and experimental (left column). The pairs differ by the coupling-field Rabi frequency (or, equivalently, by the coupling laser-field intensity $I_{c}$ ) The probe frequency was scanned across the $5 \mathrm{~S}_{1 / 2}(F=3) \rightarrow 5 \mathrm{P}_{3 / 2}\left(F^{\prime}=3\right)$ resonance, while the coupling beam frequency was kept fixed, detuned by $\Delta_{c}$ from the $5 \mathrm{P}_{3 / 2}\left(F^{\prime}=3\right)$ $\leftrightarrow 5 \mathrm{D}_{5 / 2}\left(F^{\prime \prime}=3\right)$ transition. For most of the plot pairs, the marked on the pictures detunings are $c a-8 \mathrm{MHz}$ 

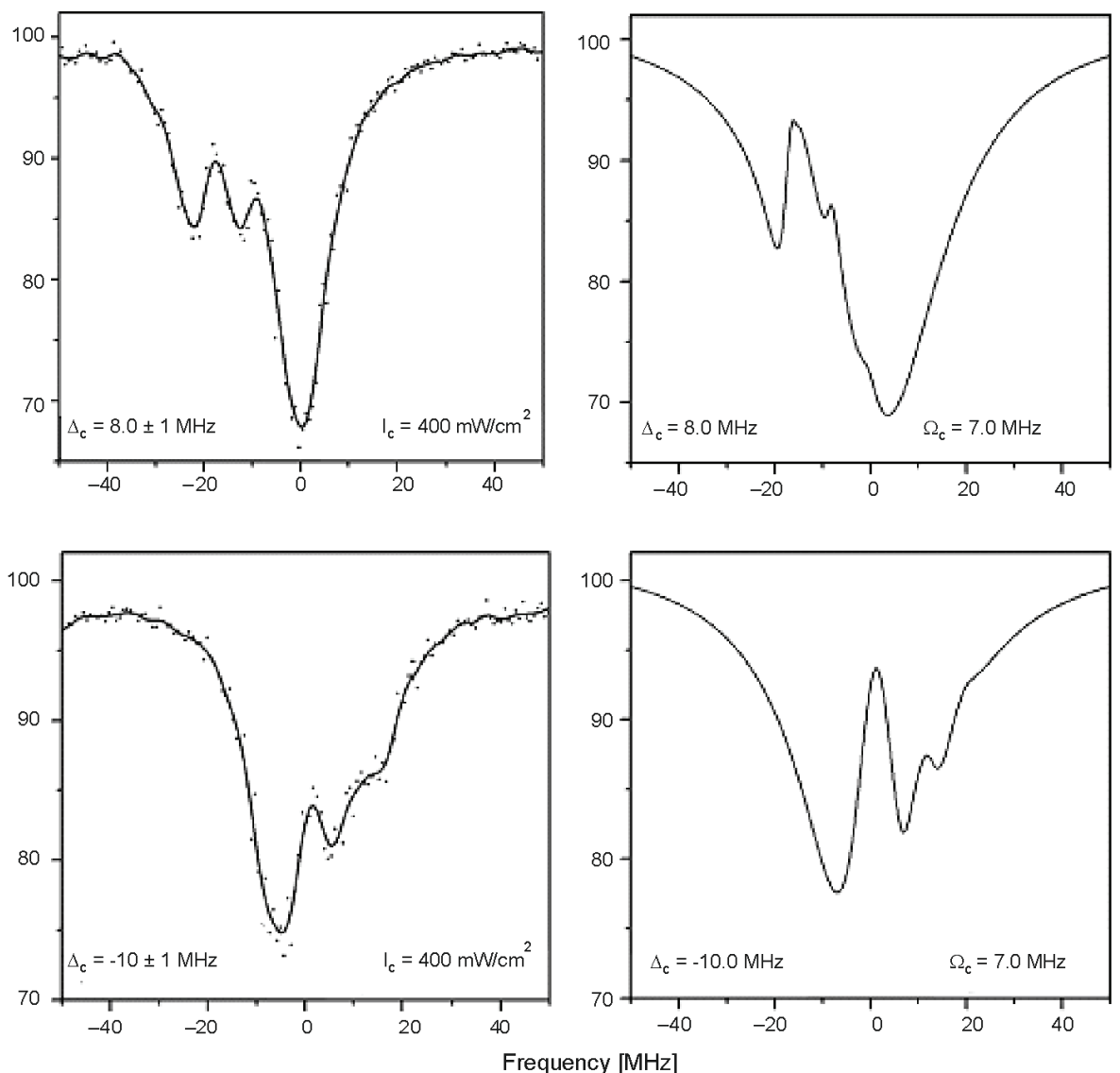

Fig. 8. EIT spectra: theoretically simulated (right column) and experimental (left column). Both experimental spectra were registered at the same coupling laser intensity, $I_{c}=400 \mathrm{~mW} / \mathrm{cm}^{2}$, for two different detunings $\Delta_{c}=+8 \mathrm{MHz}$ (upper one) and $\Delta_{c}=-10 \mathrm{MHz}$ (lower one). Spectra were registered during a short period of time when MOT was periodically switched off

pling beam off, and it contributes to the overall width of the profile with the coupling beam on (the width has to be increased in the model, as compared to the natural decay rate, e. $g$. to account for the effect of the MOT fields).

Otherwise, a good agreement between theory and experiment is reached. The EIT resonances, registered at varied experimental parameters, are well reproduced via our theoretical modeling. Some discrepancies between the model and the experimental observations may require more detailed analysis. In this mood would be an extension of the model to include the semidegenerate Zeeman substates and light polarizations. Improved control over the experimental parameters, e.g., stability of laser frequency, may be also required.

\section{SUMMARY AND CONCLUSIONS}

In the initial part of the paper (Sections II and III) we review principles and applications of the phenomenon of electromagnetically induced transparency. The phenomenon refers to the reduction of resonant absorption of a probe laser field, on an otherwise optically allowed transition, by means of a strong coupling field on a linked transition. At first, EIT was investigated mostly for atomic gas, cold or "warm". More recently the study of EIT, and of related coherent phenomena, has been extended to solids. Though solid state media are not in the mainstream of this paper, still we point out this fast developing direction in which pure as well as applied research on EIT pursues.

EIT is characterized by narrow window(s) of transparency within the absorption profile, which is accompanied by steep, positive dispersion of the refractive index. The latter property of EIT has found an application in manipulating group-velocity of light, which results in slowing, "stopping" and "storing" of light pulses, as well as in accelerating them. By reporting applications of EIT in Sect. III, we pay a particular attention to this fascinating field of research by describing some key expe- 
riments oriented on information transfer by quantum methods. We also refer to a couple of original articles and reviews.

In the second part of the paper (Sec. IV) we report our investigations of multiple windows of EIT registered in a cascade scheme $5 \mathrm{~S}_{1 / 2}(F) \rightarrow 5 \mathrm{P}_{3 / 2}\left(F^{\prime}\right) \rightarrow 5 \mathrm{D}_{J^{\prime \prime}}\left(\left\{F^{\prime \prime}\right\}\right)$ of cold ${ }^{85} \mathrm{Rb}$ atoms in MOT, where $\left\{F^{\prime \prime}\right\}$ is for a dense manifold of $h f s$ substates. Our interest in multiple-peak EIT was partially evoked by the fact that schemes providing EIT with multiple densely spaced transparency windows are seen as prospect media for slowing down simultaneously pulses of light at different nearby wavelengths. Therefore, we intended to get some insight into physics of multipeak EIT generation.

We have confirmed experimentally, in agreement with literature, that the dense $h f s$ structure of the ${ }^{85} \mathrm{Rb}\left(\mathrm{D}_{5 / 2}\right)$ state well serves to create EIT with multiple transparency windows, and their positions, shape and depth can be easily controlled by changing the coupling field intensity and/or detuning. Along with presenting experimental results of our multi-peak EIT spectra, we concentrate on a five-level model of Bloch-equations which we developed to enable theoretical interpretation of the spectra, and we compare the theory with the experiment.

A good agreement between theoretical and experimental EIT features was found. Therefore, we conclude that the applied relatively simple model of five levels and two fields, well describes the investigated EIT scheme. In view of the stated above easiness in manipulation of positions, shapes and depths of multiple EIT peaks, the present scheme should be of interest for quantum information science, e.g., by prospect manipulation of group-velocity of light via multipeak EIT. Successful theoretical reconstruction of our observed EIT features provides us with a tool for theoretical predictions in planning experiments and/or interpreting registered EIT spectra in other similar schemes. We also consider extending the model to cover more details of the scheme and of the experiment.

\section{Acknowledgements}

M. Głódź, K. Kowalski and J. Szonert gratefully acknowledge partial financial support of this work by the Polish Ministry of Science and Higher Education from the founds for science in the years 2007-2010. V. Cao Long, K. Dinh Xuan, and B. Nguyen Huy would like to thank the financial support from the Vietnam's Ministry of Science and Technology under the project coded 03/2009/HĐNĐT.

\section{References}

[1] K. Kowalski et al. Magneto-optical Trap: fundamentals and realizations. (our other paper in this issue).

[2] S.E. Harris, J.E. Field, A. Imamoglu, Nonlinear optical processes using electromagnetically induced transparency. Phys. Rev. Lett. 64, 1107 (1990).

[3] M. Fleischhauer, A. Imamoglu, J.P. Marangos, Electromagnetically induced transparency: Optics in coherent media. Rev. Mod. Phys. 77, 634 (2005).

[4] W. Gawlik, Optical nonlinearity and atomic coherences. In: Modern Nonlinear Optics, Part 3, Eds.: M. Evans, S. Kielich. Advances in Chemical Physics Series, LXXXV, 734 (1994).

[5] J.P. Marangos, Electromagnetically Induced Transparency. J. Mod. Optics 45, 471 (1998).

[6] J.P. Marangos, T. Halfmann, Electromagnetically Induced Transparency. Chapter 14 in Handbook of Optics, Third Edition, vol. IV, Optical Properties of Materials, Nonlinear Optics, Quantum Optics, Editors: M. Bass, G. Li, E.V. Stryland, Mc Graw Hill, New York etc., 14.1-14.44 (2010).

[7] A. Imamoglu, S.E. Harris, Lasers without inversion: Interference of dressed lifetime-broadened states. Opt. Lett. 14, 1344 (1989).

[8] K.J. Boller, A. Imamoglu, S.E. Harris, Observation of electromagnetically induced transparency. Phys. Rev. Lett. 66, 2593 (1991).

[9] J-J Su, I.A. Yu, The Study of Coherence-Induced Phenomena Using Double-Sided Feynman Diagrams. Chin J. Phys. 41, 627 (2003).

[10] S. Jin, Y. Li, M. Xiao, Hyperfine spectroscopy of highlyexcited atomic states based on atomic coherence. Opt. Commun. 119, 90 (1995).

[11] S. Stenholm, Foundations of Laser Spectroscopy. Wiley, N. York (1984).

[12] M. Yan, E.G. Rickey, Y. Zhu, Electromagnetically induced transparency in cold rubidium atoms. J. Opt. Soc. Am. B 18,1057 (2001).

[13] J. Gea-Banacloche, Y-q Li, S-z Jin, M. Xiao, Electromagnetically induced transparency in ladder-type inhomogeneously broadened media: Theory and experiment. Phys. Rev. A 51, 576 (1995).

[14] D.J. Fulton, S. Shepherd, R.R. Moseley, B.D. Sinclair, M.H. Dunn, Continuous-wave electromagnetically induced transparency: A comparison of $V, \Lambda$, and cascade systems. Phys. Rev. A 52, 2302 (1995).

[15] J.R. Boon, E. Zekou, D. McGloin, M.H. Dunn, Comparison of wavelength dependence in cascade, 1 , and Vee-type schemes for electromagnetically induced transparency. Phys. Rev. A 59, 4675 (1999).

[16] H.X. Chen, A.V. Durrant, J.P. Marangos, J.A. Vaccaro, Observation of transient electromagnetically induced transparency in a rubidium 1 system. Phys. Rev. A 58, 1545 (1998).

[17] J. Clarke, H. Chen, W.A. van Wijngaarden, Electromagnetically induced transparency and optical switching in a rubidium cascade system. Appl. Opt. 40, 2047 (2001).

[18] S.E. Harris, Y. Yamamoto, Photon Switching by Quantum Interference. Phys. Rev. Lett. 81, 3611 (1998).

[19] M.O. Scully, M. Fleischhauer, High-sensitivity magnetometer based on index-enhanced media. Phys. Rev. Lett. 69, 1360 (1992). 
[20] H. Lee, M. Fleischhauer, M.O. Scully, Sensitive detection of magnetic fields including their orientation with a magnetometer based on atomic phase coherence. Phys. Rev. A 58,2587 (1998)

[21] V.A. Sautenkov, M.D. Lukin, C.J. Bednar, I. Novikova, E. Mikhailov, M. Fleischhauer, V.L. Velichansky, G.R. Welch, M.O. Scully, Enhancement of magneto-optic effects via large atomic coherence in optically dense media. Phys. Rev. A 62, 023810 (2000).

[22] A.V. Turukhin, V.S. Sudarshanam, J.A. Musser, B.S. Ham, P.R. Hemmer, Observation of Ultraslow and Stored Light Pulses in a Solid. Phys.Phys. Rev. Lett. 88, 023602 (2002).

[23] B. Ham, P. Hemmer, M. Shahriar, Efficient electromagnetically induced transparency in a rare-earth doped crystal. Optics Commun. 144, 227 (1997).

[24] M. Philips, H. Wang, Electromagnetically induced transparency due to intervalence band coherence in a GaAs quantum well. Opt. Lett. 28, 831 (2003).

[25] G.B. Serapiglia, E. Paspalakis, C. Sirtori, K.L. Vodopyanov, C.C. Phillips, Observation of Laser-Induced Quantum Coherence in a Semiconductor Quantum Well. Phys. Rev. Lett. 84, 1019 (2000).

[26] M.C. Phillips, H. Wang, Spin coherence and electromagnetically induced transparency via exciton correlations. Phys. Rev. Lett. 89, 186401 (2002).

[27] W.W. Chow, H.C. Schneider, M.C. Phillips, Theory of quantum-coherence phenomena in semiconductor quantum dots. Phys. Rev. A68, 053802 (2003).

[28] M.C. Phillips, H. Wang, I. Rumyantsev, N.H. Kwong, R. Takayama, R. Binder, Electromagnetically Induced Transparency in Semiconductors via Biexciton Coherence. Phys. Rev. Lett. 91, 183602 (2003).

[29] J. Houmark, A.P. Jauho, T.R. Nielsen, J. Mørk, Influence of many-particle interactions on slow light phenomena in quantum dots. J. Phys. Conference Series 107, 012005 (2008).

[30] Y. Wu, X. Yang, Phys. Rev. A 71, 053806 (2005).

[31] S. Marcinkievicius, A. Gushterov, J.P. Reithmaier, Transient electromagnetically induced transparency in self-assembled quantum dots. Appl. Phys. Lett. 92, 041113 (2008).

[32] Y. Okawachi, M.S. Bigelow, J.E. Sharping, Z. Zhu, A. Schweinsberg, D.J. Gauthier, R.W. Boyd, A.L. Gaeta, Tunable All-Optical Delays via Brillouin Slow Light in an Optical Fiber. Phys. Rev. Lett. 94, 153902 (2005).

[33] Q. Xu, S. Sandhu, M.L. Povinelli, J. Shakya, S. Fan, M. Lipson, Experimental Realization of an On-Chip All-Optical Analogue to Electromagnetically Induced Transparency. Phys. Rev. Lett. 96, 123901 (2006).

[34] H. Gersen, T.J. Karle, R.J.P. Engelen, W. Bogaerts, J.P. Korterik, N.F. van Hulst, T.F. Krauss, L. Kuipers, RealSpace Observation of Ultraslow Light in Photonic Crystal Waveguides. Phys. Rev. Lett. 94, 073903 (2005).

[35] S.E. Mingaleev, A.E. Miroshnichenko, Y.S. Kivshar, $\mathrm{K}$. Busch, All-optical switching, bistability, and slow-light transmission in photonic crystal waveguide-resonator structures. Phys Rev E 74, 046603-(1-15) (2006).

[36] A. Rostami, G. Rostami, Full-optical realization of tunable low pass, high pass and band pass optical filters using ring resonators. Opt. Commun. 240, 133 (2004).

[37] M. Davanco, P. Holmstrom, D.J. Blumenthal, L. Thylen, Directional coupler wavelength filters based on waveguides exhibiting electromagnetically induced transparency. IEEE J. Quant. Eelectr. 39 (4) (2003).
[38] A. Neogi, T. Mozume, H. Yoshida, O. Wada, Intersubband transition at 1.3 and $1.55 \mathrm{~lm}$ in a novel coupled InGaAs/AlAsSb quantum well structures. IEEE Photon. Technol. Lett. 11, 632 (1999).

[39] M.D. Lukin, Trapping and manipulating photon states in atomic ensembles. Rev. Mod. Phys. 75, 457 (2003).

[40] S.E. Harris, J.E. Filed, A. Kasapi, Dispersive properties of electromagnetically induced transparency. Phys Rev. A 46, 29 (1992).

[41] A. Kasapi, M. Jain, G.Y. Yin, S.E. Harris, Electromagnetically Induced Transparency: Propagation Dynamics. Phys. Rev. Lett. 74, 2447 (1995)

[42] O. Schmidt, R. Wynands, Z. Hussein, D. Meschede, Steep dispersion and group velocity below c/3000 in coherent population trapping. Phys. Rev. A 53, R27 (1996).

[43] L.V. Hau, S.E. Harris, Z. Dutton, C.H. Behroozi, Light speed to 17 metres per second in an ultracold atomic gas. Nature 397, 594 (1999).

[44] M.M. Kash, V.A. Sautenkov, A.S. Zibrov, L. Hollberg, George R. Welch, M.D. Lukin, Y. Rostovtsev, E.S. Fry, M.O. Scully, Ultraslow Group Velocity and Enhanced Nonlinear Optical Effects in a Coherently Driven Hot Atomic Gas. Phys. Rev. Lett. 82, 5229 (1999).

[45] D. Budker, F. Kimball, S.M. Rochester, V.V. Yashchuk, Nonlinear Magneto-optics and Reduced Group Velocity of Light in Atomic Vapor with Slow Ground State Relaxation. Phys. Rev. Lett. 83, 1767 (1999).

[46] D.F. Phillips, A. Fleischhauer, A. Mair, R.L. Walsworth, Storage of Light in Atomic Vapor. Phys. Rev. Lett. 85, 783 (2001).

[47] Ch. Liu, Z. Dutton, C.H. Behroozi, L.V. Hau, Observation of coherent optical information storage in anatomic medium using halted light pulses. Nature 409, 493 (2001).

[48] Slow light. Nature Photonics, Focus issue. 447-509 (August 2008, vol. 2), collection of articles.

[49] M.D. Lukin, Colloquium: Trapping and manipulating photon states in atomic ensemble. Rev. Mod. Phys. 75, 457-472 (2003).

[50] Zaremba, Propagation and storing of light in optically modified atomic media. J. Phys.: Conf. Ser. 213 012025-110 (2010).

[51] M. Fleischhauer, M.D. Lukin, Quantum memory for photons: Dark-state polaritons. Phys. Rev. A, 65, 022314 (2002).

[52] D. Dziczek, B. Ziętek, S. Chwirot, Low-Speed and Suspended Propagation of Light Pulses in Atomic Medium with Electromagnetically Induced Transparency. Acta Phys. Pol. A 106, 13 (2004).

[53] D. Dziczek, S. Chwirot, Dual control of slow light in reciprocal electromagnetically-induced-transparency conditions. Phys. Rev. A 79, 043807-1-9 (2009).

[54] A.V. Gorshkov, A. André, M.D. Lukin, A.S. Sørensen, Photon storage in A-type optically dense atomic media. III. Effects of inhomogeneous broadening. Phys. Rev. A 76, 033806-1-13 (2007).

[55] N.S. Ginsberg, S.R. Garner, L.V. Hau, Coherent control of optical information with matter wave dynamics. Nature 445, 623 (2007).

[56] T. Baba, D. Mori, Slow light engineering in photonic crystals. J. Phys. D 40 2659-2665 (2007).

[57] G.M. Gehring, R.W. Boyd, A.L. Gaeta, D.J. Gauthier, Fellow, A.E. Willner, Fiber-Based Slow-Light Technologies. Journal of Lightwave Technologies 23, 3752-3762 (2008).

[58] T.F. Krauss, Slow light in photonic crystal waveguides. J. Phys. D 40, 2666-2670 (2007). 
[59] C. Monat, M. de Sterke, B.J. Eggleton, Slow light enhanced nonlinear optics in periodic structures. J. Opt. 12, $104003-$ $1-17(2010)$

[60] S.A. Schulz, L.O. Faolain, D.M. Beggs, T.P. White, A. Melloni, T.F. Krauss, Dispersion engineered slow light in photonic crystals: a comparison. J. Opt. 12, 104004-1-10 (2010).

[61] K. Kowalski, E. Dimova-Arnaudova, K. Fronc, S. Gateva, M. Głódź, L. Lis, L. Petrov, J. Szonert, A System for magneto-optical cooling and trapping of $R b$ atoms. Opt. Applicata 36, 559 (2006).

[62] K. Kowalski, K. Vaseva, S. Gateva, M. Głódź, L. Petrov, J. Szonert, System for EIT spectroscopy of cold Rb atoms. Proc. of SPIE 6604 (2007) 66040K-1-5 (2007).

[63] K. Kowalski, V. Cao Long, H. Nguyen Viet, S. Gateva, M. Głódź, J. Szonert, Simultaneous coupling of three hfs components in a cascade scheme of EIT in cold $85 R \mathrm{~B}$ atoms. J. Non-Cryst. Solids 355, 1295-1301 (2009).

[64] Cao Long Van, Khoa Dinh Xuan, Thuan Bui Dinh, Hung Nguyen Viet, EIT in multi-level cascade scheme of cold rubidium atoms: theoretical conciderations. Communications in Physics 18, 146-150 (2008).

[65] J. Wang, L.B. Kong, X.H. Tu, K.J. Jiang, K. Li, H.W. Xiong, Y.F. Zhu, M.S. Zhan, Electromagnetically induced trans- parency in multi-level cascade scheme of cold rubidium atoms. Phys. Lett. A 328, 437 (2004).

[66] S.R. Echaniz, A.D. Greentree, A.V. Durrant, D.M. Segal, J.P. Marangos, J.A. Vacaro, Observations of a doubly driven $V$ system probed to a fourth level in laser-cooled rubidium. Phys. Rev. A 013812 (2001).

[67] D. McGloin, Coherent effects in a driven Vee scheme. Phys. B 36, 2861 (2003)

[68] D. McGloin, D.J. Fulton, M.H. Dunn, Electromagnetically induced transparency in $\mathrm{N}$-level cascade scheme. Opt. Commun. 190, 221 (2001).

[69] J. Wang, Y. Zhu, K.J. Jiang, M.S. Zhan, Bichromatic electromagnetically induced transparency in cold rubidium atoms. Phys. Rev. A 68, 063810 (2003).

[70] X-M. Hu, G-L. Cheng, J-H. Zou, X. Li, D. Du, Double switching from normal to anomalous dispersion via trichromatic phase manipulation of electromagnetically induced transparency. Phys. Rev. A72, 023803 (2005).

[71] M.O. Scully, M.S. Zubairy, Quantum Optics, Cambridge University Press, 1997.

[72] Y.C. Chen, C.-W. Lin, I.A. Yu, Roles of degenerate Zeeman levels in electromagnetically induced transparency. Phys. Rev. A 61, 053805 (2000).
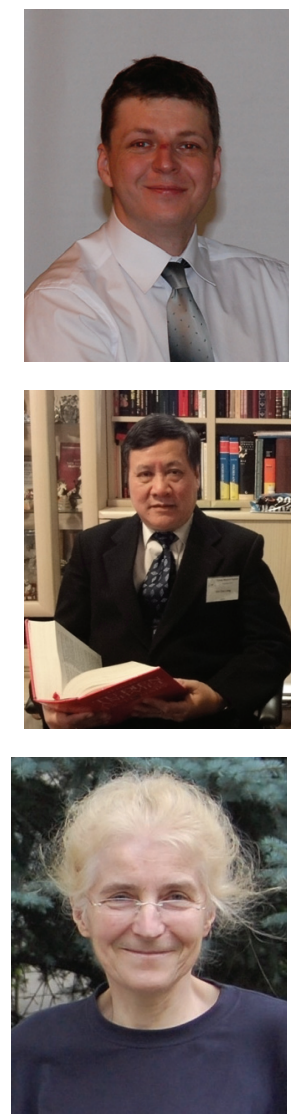

KRZYSZTOF KowALSKI, has been graduated from the College of Science in Warsaw with master degree in experimental physics. His fields of interest cover cold atom physics, quantum and nonlinear optics and the design of digital measrement systems. He is the builder of Warsaw magneto-optical trap. In 2008 he defended his PhD thesis: "Electromagnetically Induced Transparency in cold rubidium atoms in magneto-optical trap" at the Institute of Physics PAS, supervised by Assoc. Prof. Małgorzata Głódź.

CaO Long VAN was born in 1952 in Hanoi, Vietnam. He graduated from Warsaw University in 1976 with an MSc degree in theoretical physics. His master thesis was entitled "Callan-Symanzik Equation and Transition Probabilities" and was written under the supervision of Prof. Iwo Białynicki-Birula. In 1979, he defended his $\mathrm{PhD}$ dissertation entitled "Phase Representation in Quantum Optics", also supervised by Prof. I. BiałynickiBirula. He habilitated in 1987 in Center of Theoretical Physics, Polish Academy of Sciences in Warsaw with a thesis entitled "Noises in Quantum Optics". His research interests concern several topics in Quantum and Nonlinear Optics.

Matgorzata Gıódź received MSc degree from the Warsaw University and she gained her PhD and habilitation at the Institute of Physics of the Polish Academy of Sciences (IP PAS) in Warsaw. She is working at IP PAS at a position equivalent to Assoc. Prof. For two years she was also doing research in Canada, as a guest of the Department of Physics of the University of Windsor. Her main scientific interests concentrate on atom-laser-field interactions. She dealt with experiments on the statistical properties of laser light and HiRes (quantum-beat) $h f s$ spectroscopy, in collaboration with the Department of Physics of the Warsaw University. In Canada, and then also in Poland, she studied atomic deexcitation processes, spontaneous and in thermal collisions. Currently, she is investigating cold atoms (coherent processes, HiRes spectroscopy) in a magnetooptical trap. 

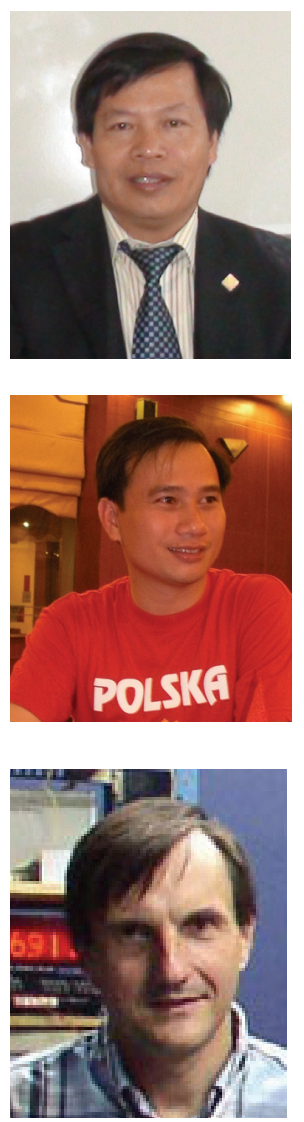

DinH XuAn KhOA was born in Nghe An province, Vietnam. He graduated, with a major in Physics, from Vinh University in 1981. He completed a PhD course in Quantum Optics in 1996. His doctoral thesis entitled "Physics of Dye Lasers" was supervised by Prof. Cao Long Van and Prof. Dao Xuan Hoi. Since 1997 he has been the head of research group of Optics in Vinh University. He was elected as associate professor in Physics in 2003. His field of interests covers a large variety of topics in Quantum and Nonlinear Optics, Atomic and molecular Physics.

NGUYEN HUy BANG was born in Ha Tinh province, Vietnam. He graduated, in physics, from Vinh University in 2002. In 2004 he did PhD study at Institute of Physics, Polish Academy of Sciences, under the supervision of Prof. W. Jastrzebski. Here he did research on alkali-metal diatomic molecules by using polarization labelling spectroscopy technique. After his graduation in Poland in 2008, he returned to work as lecturer in Optics group, faculty of Physics, Vinh University. Presently, his field of interest concerning to physics of atoms, molecules, and optics.

JERZY SzONERT graduated from Warsaw University with a MSc degree in experimental physics. His field of interest covers various topics of Spectroscopy, Nonlinear and Quantum Optics. Currently he is involved in experiments on cold atoms in the group of Assoc. Prof. Małgorzata Głódź. He is working in the Institute of Physics, Polish Academy of Sciences in Warsaw. 